\title{
THREE CHARACTERISTIC CLASSES MEASURING THE OBSTRUCTION TO PL LOCAL UNKNOTEDNESS
}

\author{
BY LOWELL EDWIN JONES
}

Communicated by S. S. Chern, May 12, 1972

$(M, \partial M) \subset(N, \partial N)$ denotes a PL embedding of oriented PL manifolds, with $M \cap \partial N=\partial M, \operatorname{codim}_{N}(M)=2$. Two such embeddings $i_{0}, i_{1}$ are concordant if there exists a PL embedding $(M \times I, \partial M \times I, M \times \partial I) \subset$ $(N \times I, \partial N \times I, N \times \partial I)$ restricting to $i_{0}, i_{1}$ on $M \times 0, M \times 1$, respectively. The embedding $(M, \partial M) \subset(N, \partial N)$ is locally flat if every point $x \in M$ has a PL ball $D^{n}$ for its neighborhood such that $D^{n} \cap M \subset D^{n}$ is PL conjugate to the standard embedding $D^{n-2} \subset D^{n}$.

This is the problem considered here: When is the embedding $(M, \partial M) \subset$ $(N, \partial N)$ concordant to a locally flat embedding?

Let $G_{k}$ denote the (geometric) group of concordism classes of locally flat knots having dimension $k$. Stabilizing, and factoring out by the periodicity isomorphism, we get a $Z_{4}$-graded group, which is denoted (somewhat akwardly) as $G_{*}$. $K_{F / P L}^{*}, K_{F / T O P}^{*}$ denote the cohomology theories having $F / P L, \overline{F / T O P}$ for their zeroth loop spectrum [11].

THEOREM. There are characteristic classes $\theta(M, N) \in H^{2}\left(M, G_{3}\right), \beta(M, N)$ $\in K_{F / P L}^{0}(M), \gamma(M, N) \in K_{F / T O P}^{0}\left(M, G_{*+1}\right)$ satisfying the following:

(a) These classes depend only on the concordism class of the embedding $(M, \partial M) \subset(N, \partial N)$.

(b) They vanish if and only if $(M, \partial M) \subset(N, \partial N)$ is concordant to a locally flat embedding.

Construction of $\theta, \beta, \gamma$.

$\theta$. For each simplex $\Delta^{k} \in M$ there are cells $D_{M}\left(\Delta^{k}\right), D_{N}\left(\Delta^{k}\right)$-the dual cells to $\Delta^{k}$ in $M, N$, respectively. These satisfy $D_{N}\left(\Delta^{k}\right) \cap M=D_{M}\left(\Delta^{k}\right)$; $D_{m}\left(\Delta^{k}\right) \subset D_{N}\left(\Delta^{k}\right)$ is a codimension 2 embedding of discs. Try to concord $(M, \partial M) \subset(N, \partial N)$ to a locally flat embedding by inductively doing so for the embeddings $\left(D_{M}\left(\Delta^{k}\right) \subset D_{N}\left(\Delta^{k}\right)\right.$. The first possible nonvanishing obstruction appears as a cocycle defined on the 2-dimensional dual cells in $M$. This represents $\theta(M, N) \in H^{2}\left(M, G_{3}\right)[10]$.

$\gamma$. Let $\left(R, R_{\partial}\right)$ denote a regular neighborhood for $(M, \partial M) \subset(N, \partial N)$. $\dot{R}$ denotes its topological boundary in $N$. There is a linear bundle $\tau$ defined over $M$, having $D^{2}$ for fiber, and an integral-homology equivalence 
$f:(R, \dot{R}) \rightarrow(\tau, \dot{\tau})$. Using $f$ in conjunction with the new surgery groups of Cappell-Shaneson, a splitting obstruction can be defined along any singular $Z_{q}$-manifold $g:(V, \delta V) \rightarrow M$ as in [6]. By the universal coefficient theorem for $K_{F / \text { TOP }}^{*}$, these represent an element $\gamma(M, N) \in K_{F / T O P}^{0}\left(M, G_{*+1}\right)$.

$\beta$. For large $r, f \times \dot{1}_{D^{r}}:(R, \dot{R}) \times\left(D^{r}, \partial D^{r}\right) \rightarrow(\tau, \dot{\tau}) \times\left(D^{r}, \partial D^{r}\right)$ represents an element $\beta(M, N) \in K_{F / P L}^{0}(M)$.

REMARK 1. There is a classifying space $B S P L^{\sim}(2)$ for codimension 2 PL thickenings of PL manifolds. There are universal characteristic classes

$$
\begin{aligned}
& \theta \in H^{2}\left(B S P L^{\sim}(2), G_{3}\right), \\
& \beta \in K_{F / P L}^{0}\left(B S P L^{\sim}(2)\right), \\
& \gamma \in K_{\text {F/TOP }}^{0}\left(B S P L^{\sim}(2), G_{*+1}\right) .
\end{aligned}
$$

Modulo low-dimensional complications (over the 4 skeleton of $B S P L^{\sim}$ (2)) there is a homotopy equivalence

$$
B S P L \sim(2) \stackrel{f \times y}{\longrightarrow} B S O(2) \times\left(\overline{F / T O P} \otimes G_{*+1}\right) .
$$

REMARK 2. The classes $\theta, \beta, \gamma$ are related as follows. The geometric stabilization of $\theta$ is determined by the restriction of $\gamma$ to the 2-skeleton of $M$. The stabilization of $\beta$ is a direct summand of $\gamma$.

\section{BIBLIOGRAPHY}

1. S. Cappell and J. Shaneson, Submanifolds, group actions, and knots. I, II, Princeton University, 1972 (mimeographed notes).

2. M. A. Kervaire, Les nouds de dimensions supérieures, Bull. Soc. Math. France 93 (1965), 225-271. MR 32 \# 6479.

3. J. Levine, Invariants of knot cobordism, Invent. Math. 8 (1969), 98-110; addendum, ibid., 355. MR 40 \# 6563 .

4. C. P. Rourke and B. J. Sanderson, Block bundles. I, II, III, Ann. of Math. (2) 87 (1968), 1-28, 256-278, 431-483. MR 37 \# 2234a,b; MR 38 \# 729.

5. D. Sullivan, Thesis, Princeton University, Princeton, N.J., 1966.

6. $\longrightarrow$, Geometric topology, Princeton University, 1967 (mimeographed notes).

7. J. .Wagoner, Thesis, Princeton University, Princeton, N.J., 1966.

8. C. T. C. Wall, Surgery on compact manifolds, Academic Press, New York, 1971.

9. - Locally flat PL submanifolds with codimension two, Proc. Cambridge Philos. Soc. 63 (1967), 5-8. MR 37 \#3577.

10. H. Noguchi, Obstructions to locally flat embeddings of combinatorial manifolds, Topology 5 (1966), 203-213.

11. L. Jones, Combinatorial symmetries of $D^{m}$. III, Berkeley, California, 1971 (lecture notes).

Department of Mathematics, University of California, Berkeley, California 94720

Current address: Department of Mathematics, Princeton University, Princeton, New Jersey 08540 